Sādhanā Vol. 37, Part 4, August 2012, pp. 481-492. (C) Indian Academy of Sciences

\title{
Degree of coupling in high-rise mixed shear walls structures
}

\author{
J C D HOENDERKAMP
}

Department of the Built Environment, Eindhoven University of Technology, 5600MB Eindhoven, The Netherlands

e-mail: j.c.d.hoenderkamp@tue.nl

MS received 21 April 2011; accepted 2 April 2012

\begin{abstract}
A simple method of analysis is presented to determine the influence of single shear walls (SSW) on the degree of coupling $D o C$ and on the peak shear demand PSD for beams of coupled shear walls (CSW) in mixed shear wall structures (MSW). Non-coupled lateral load resisting structures such as singular planar walls and cores will reduce primary bending moments in the coupled shear wall bents of MSW structures thereby increasing the degree of coupling. They will also change the location and magnitude of the maximum shear in and rotation of the coupling beams. These changes in the coupled wall bents may increase the demand on their performance beyond capacity. It is, therefore, important to have an indication of the change in the coupling beam design parameters at an early stage of the design. The proposed graphical method is based on the continuous medium theory and allows a rapid assessment of the structural behaviour of coupled shear wall bents in mixed shear wall structures that are subject to horizontal loading.
\end{abstract}

Keywords. Coupled shear walls; degree of coupling; peak shear demand; concrete.

\section{Introduction}

In reinforced concrete tall buildings, coupled wall structures as shown in figure 1 can provide an efficient lateral load resisting system against wind and earthquake effects. When such structures are subjected to horizontal loading, the total overturning moment is divided into two components: primary bending moments, $M_{1}$ and $M_{2}$ taken by the individual walls and an axial bending moment $T \ell$ which is the axial force, $T$ induced in the walls by the shear forces in the coupling beams, multiplied by a distance $\ell$ between the neutral axes of the walls.

The relationship between these two types of bending moment can be represented by a single parameter. The degree of coupling $(D o C)$ of coupled shear walls (CSW) is defined by the axial bending moment at the base of the structure, expressed as a fraction of the total overturning moment

$$
D o C=\frac{T \ell}{T \ell+M_{1}+M_{2}} .
$$




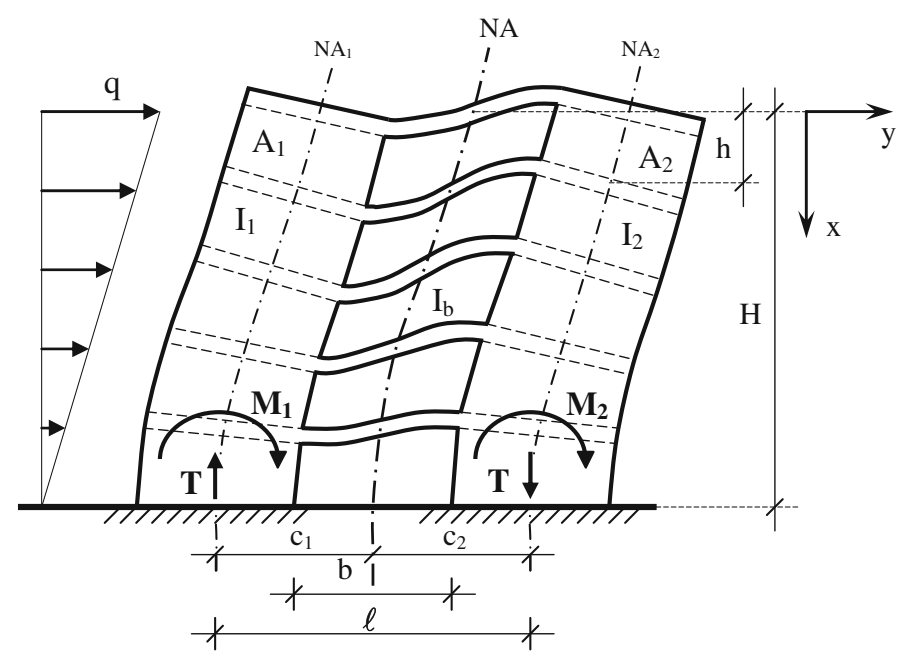

Figure 1. Coupled shear wall structure.

Obtaining the different types of bending moment requires a full analysis of the structure as the relationship between these actions is dependent on the stiffness of the coupling beams and is not constant over the height of the structure. The continuous medium theory as applied to high-rise coupled shear wall structures (Chitty 1947) allows a rapid assessment of the horizontal deflections when subjected to lateral loading (Coull \& Choudhury 1967; Stafford Smith et al 1981; Stafford Smith \& Coull 1991). The theory was extended to allow the addition of other types of bents to be included in the deflection analysis such as single shear walls and rigid frames in multi-bent structures (Stafford Smith et al 1982, 1984). The calculation of the overturning moments was also made possible (Hoenderkamp \& Stafford Smith 1984) which allowed expressions to be derived for the shear forces in the coupling beams in MSW structures. Establishing the maximum shear force, $V_{\mathrm{b}, \max }$ in the critical coupling beam and its location up the height of the structure will yield a relationship between this peak shear force and an average shear force, $V_{\mathrm{b}, \text { avg }}$ which is called the peak shear demand (PSD) of a coupling beam

$$
P S D=\frac{V_{\mathrm{b} \cdot \max }}{V_{\mathrm{b}, \mathrm{avg}}} .
$$

The degree of coupling (DoC) and the peak shear demand (PSD) are two very important parameters in the design of coupled wall structures (Chaallal et al 1996).

\section{Coupled shear wall structures}

For a coupled wall structure subject to a triangularly distributed lateral load with a maximum value of $q$ at the top and zero at the base of the structure, the degree of coupling is (Harries et al 2004) given as

$$
D o C_{\mathrm{csw}}=\frac{T \ell}{q H^{2} / 3}=\frac{3}{k^{2}}\left\{\frac{1}{3}-\frac{1-(1 / k \alpha H-k \alpha H / 2) \sinh (k \alpha H)}{(k \alpha H)^{2} \cosh (k \alpha H)}\right\},
$$


where $H$ is the total height of the structure and a characteristic structural parameter

$$
\alpha^{2}=\frac{G A}{E I},
$$

in which $G A$ is the racking stiffness of the structure and $E I$ is its flexural stiffness. The flexural stiffness of a coupled wall bent comprising two shear walls as shown in figure 1 is the sum of the individual bending stiffness of the walls

$$
E I_{\mathrm{csw}}=E I_{1}+E I_{2}
$$

A characteristic non-dimensional parameter is expressed as follows

$$
k^{2}=\frac{E I+E A c^{2}}{E A c^{2}},
$$

where $E A c^{2}$ is the total axial bending stiffness of the structure. The axial bending stiffness of a coupled wall bent is given by

$$
E A c_{\mathrm{csw}}^{2}=\Sigma E A_{\mathrm{i}} c_{\mathrm{i}}^{2},
$$

in which $c_{\mathrm{i}}$ is the distance between the neutral axis of an individual shear wall and the overall neutral axis of the coupled wall bent as shown in figure 1 . The racking shear stiffness of a coupled wall structure is given by

$$
G A_{\mathrm{csw}}=\frac{12}{h\left\{\frac{h}{\gamma^{3} E I_{\mathrm{csw}}}+\frac{\ell}{\beta^{3} E I_{\mathrm{b}}}\right\}},
$$

in which two non-dimensional parameters are defined as follows

$$
\beta=(\ell / b),
$$

where $b$ is the length of the flexible coupling beams between the coupled walls,

$$
\gamma=\{h /(h-d)\}
$$

in which $d$ is the depth of the coupling beams and $h$ is the story height.

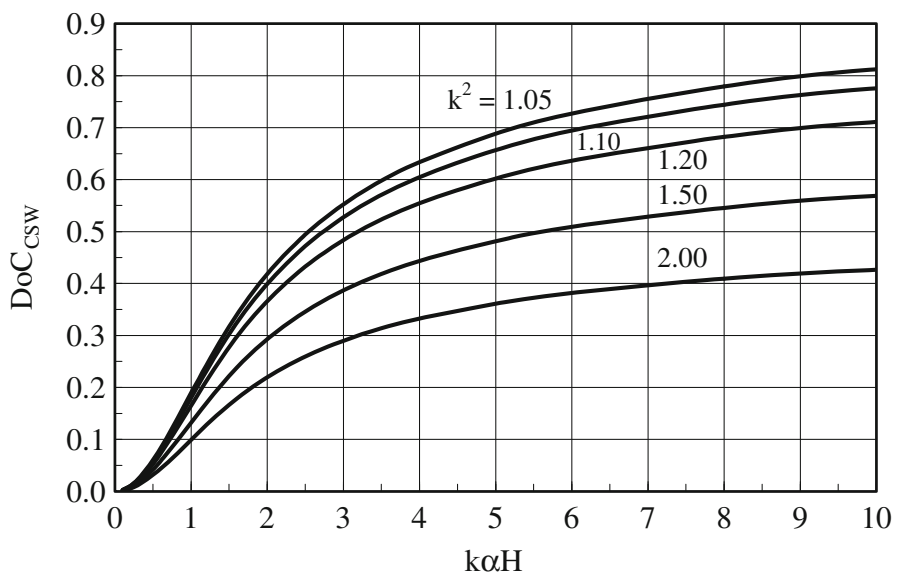

Figure 2. Degree of coupling for coupled wall structures subject to triangularly distributed loading. 
The diagram in figure 2 allows a rapid assessment of the degree of coupling in coupled shear wall structures as a function of two non-dimensional characteristic parameters, $k \alpha H$ and $k^{2}$.

\section{Mixed shear wall structures}

\subsection{Continuous medium approach}

Adding single shear walls or cores to coupled shear wall structures or replacing coupled shear wall bents with single shear walls as shown in figure 3, will alter the load distribution between the various lateral load resisting elements. A coupled wall structure has three characteristic structural parameters: bending stiffness $E I$, racking shear stiffness $G A$ and axial bending stiffness $E A c^{2}$ which define its behaviour when subjected to horizontal loading. The lateral deflection behaviour of a single shear wall can be well-represented by its bending stiffness EI. It has been shown (Stafford Smith et al 1982, 1984) that the horizontal deflections up the height of a high-rise mixed shear wall structure (MSW) can be expressed as follows

$$
\begin{aligned}
y= & \frac{q H^{4}}{E I_{\mathrm{g}}}\left[\frac{11}{120}-\frac{1}{8}\left(\frac{x}{H}\right)+\frac{1}{24}\left(\frac{x}{H}\right)^{4}-\frac{1}{120}\left(\frac{x}{H}\right)^{5}\right. \\
& +\frac{1}{k^{2}-1}\left\{\frac{1}{(k \alpha H)^{2}}\left(\frac{1}{3}-\frac{1}{2}\left(\frac{x}{H}\right)^{2}+\frac{1}{6}\left(\frac{x}{H}\right)^{3}-\frac{1-x / H}{(k \alpha H)^{2}}\right)\right. \\
& \left.\left.+\frac{\cosh (k \alpha H)(1-x / H)-1+\{1 /(k \alpha H)-(k \alpha H) / 2\}\{\sinh (k \alpha H)-\sinh (k \alpha x)\}}{(k \alpha H)^{4} \cosh (k \alpha H)}\right\}\right],
\end{aligned}
$$

in which the gross bending stiffness of the structure is the sum of all primary and axial bending stiffness in the mixed shear wall structure

$$
E I_{\mathrm{g}}=\Sigma E I_{\mathrm{csw}}+\Sigma E I_{\mathrm{ssw}}+\Sigma E A c^{2}
$$

and $x$ is the distance measured from the top of the structure. Equations (4) and (6) can also be used here to obtain the characteristic parameters $\alpha^{2}$ and $k^{2}$ by summing for all stiffness components in the mixed shear wall structure. The individual primary bending moments in the wall can be obtained from the second derivative of equation (11) which must be multiplied by the

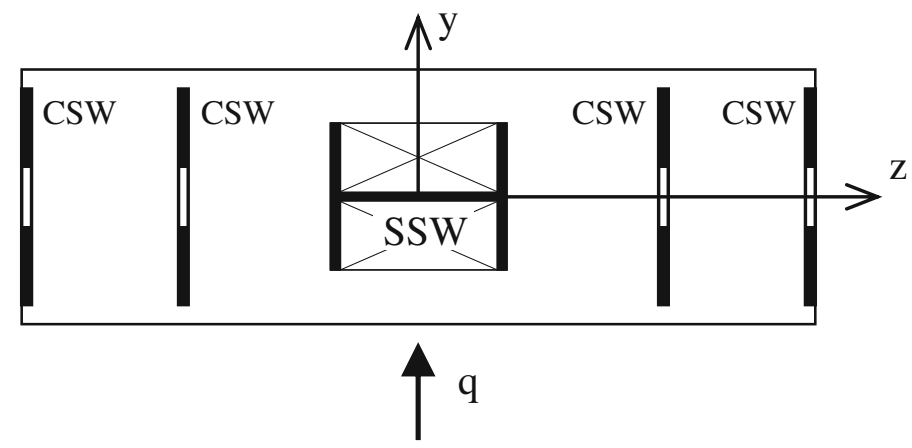

Figure 3. Typical floor plan of mixed shear wall structure. 
individual bending stiffness of the wall (Hoenderkamp \& Stafford Smith 1984). The second derivative is given by

$$
\begin{aligned}
y^{\prime \prime}= & \frac{q H^{2}}{E I_{\mathrm{g}}}\left[\frac{1}{2}\left(\frac{x}{H}\right)^{2}-\frac{1}{6}\left(\frac{x}{H}\right)^{3}+\frac{1}{k^{2}-1}\left\{-\frac{1-x / H}{(k \alpha H)^{2}}\right.\right. \\
& \left.\left.+\frac{\cosh (k \alpha H)(1-x / H)-\{1 /(k \alpha H)-(k \alpha H) / 2\} \sinh (k \alpha x)}{(k \alpha H)^{2} \cosh (k \alpha H)}\right\}\right] .
\end{aligned}
$$

For a coupled wall structure the primary bending moments in the two walls are

$$
M_{1}+M_{2}=E I_{\mathrm{csw}} y^{\prime \prime}
$$

The total axial bending moment (Hoenderkamp \& Stafford Smith 1984) in the structure is

$$
\begin{aligned}
T \ell= & \frac{q H^{2}}{k^{2}}\left[\frac{1}{2}\left(\frac{x}{H}\right)^{2}-\frac{1}{6}\left(\frac{x}{H}\right)^{3}+\frac{1-x / H}{(k \alpha H)^{2}}\right. \\
& \left.-\frac{\cosh (k \alpha H)(1-x / H)-\{1 /(k \alpha H)-(k \alpha H) / 2\} \sinh (k \alpha x)}{(k \alpha H)^{2} \cosh (k \alpha H)}\right] .
\end{aligned}
$$

This bending moment is distributed to each coupled wall bent in the MSW structure according to its individual axial bending stiffness, i.e.,

$$
T \ell_{\mathrm{csw}}=T \ell \frac{E A c_{\mathrm{csw}}^{2}}{E A c^{2}}
$$

The degree of coupling for a specific coupled shear wall bent can be obtained by evaluating the primary and axial bending moments at the base of the structure, where $x=H$. Then, using equation (1) will yield

$$
D o C=\frac{T \ell_{\mathrm{csw}}}{E I_{\mathrm{csw}} y^{\prime \prime}+T \ell_{\mathrm{csw}}}=\left[\frac{E I_{\mathrm{csw}} y^{\prime \prime}}{T \ell_{\mathrm{csw}}}+1\right]^{-1} .
$$

Substituting equations (6), (13), (14), (15) and (16) into equation (17) and simplifying leads to the following expression for the degree of coupling

$$
D o C=\left\{1+\left(k_{\mathrm{csw}}^{2}-1\right)\left(\frac{1+C /\left(k^{2}-1\right)}{1-C}\right)\right\}^{-1},
$$

in which the constant $C$ for a triangularly distributed lateral load (TDL) is given by

$$
C_{\mathrm{TDL}}=3\left\{\frac{1-(1 / k \alpha H-k \alpha H / 2) \sinh (k \alpha H)}{(k \alpha H)^{2} \cosh (k \alpha H)}\right\} .
$$

A characteristic structural parameter for a specific coupled wall bent for which the degree of coupling is to be obtained, can be expressed as follows

$$
k_{\mathrm{csw}}^{2}=\frac{E I_{\mathrm{csw}}+E A c_{\mathrm{csw}}^{2}}{E A c_{\mathrm{csw}}^{2}} .
$$




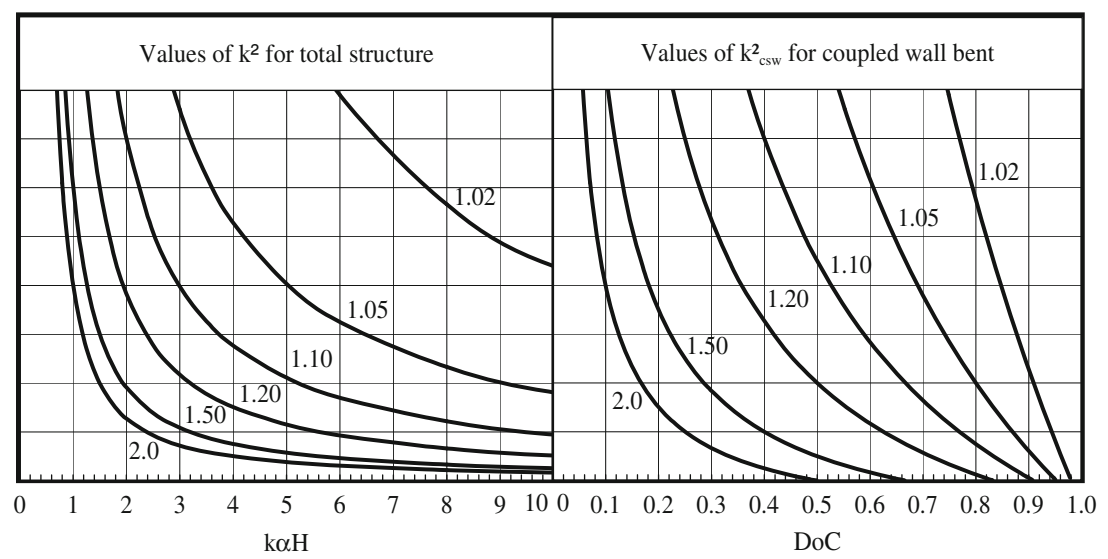

Figure 4. Degree of coupling for mixed structures subject to triangularly distributed horizontal loads.

For a rapid assessment of the $D o C$ of a coupled wall bent in a mixed shear wall structure, the diagram in figure 4 is entered on the left hand side of the graph with values for $k \alpha H$ and $k^{2}$ which represent the mixed shear wall structure. From a curve on the left hand side, the right hand side is entered with a value for $k_{\mathrm{csw}}^{2}$ for the specific coupled wall bent. Figure 4 can also be used for structures consisting of coupled shear wall bents only, i.e., $k^{2}=k_{\mathrm{csw}}^{2}$.

\section{Coupling beam shear capacity}

\subsection{Maximum shear force in coupling beam and its location}

The change in axial force in an individual wall of a coupled wall bent represents the shear in the beams connecting the walls (Coull \& Choudhury 1967) and is given by the first derivative of equation (15)

$$
\begin{aligned}
T^{\prime} \ell= & \frac{q H}{k^{2}}\left[\left(\frac{x}{H}\right)-\frac{1}{2}\left(\frac{x}{H}\right)^{2}-\frac{1}{(k \alpha H)^{2}}\right. \\
& \left.-\frac{-\sinh (k \alpha H)(1-x / H)-(1 / k \alpha H-k \alpha H / 2) \cosh (k \alpha x)}{(k \alpha H) \cosh (k \alpha H)}\right] .
\end{aligned}
$$

The shear in the connecting beams of all the coupled walls in the structure is distributed to the beams of a specific coupled wall bent according to its axial bending stiffness

$$
T^{\prime} \ell_{\mathrm{csw}}=T^{\prime} \ell \frac{E A c_{\mathrm{csw}}^{2}}{E A c^{2}}
$$

The location of the maximum shear is obtained by differentiating equation (21) with respect to $x$, setting it equal to zero and solving for $x / H$

$$
T^{\prime \prime} \ell=\frac{q}{k^{2}}\left[1-\left(\frac{x}{H}\right)-\frac{\cosh (k \alpha H)(1-x / H)-(1 / k \alpha H-k \alpha H / 2) \sinh (k \alpha x)}{\cosh (k \alpha H)}\right]=0 .
$$


This equation is only dependent on the characteristic parameter $k \alpha H$. Figure 5 shows a graphical solution to equation (23) for values of $x / H$. This location of maximum shear force in the connecting beam is not the same as for the maximum slope in the structure. The location of maximum story sway is a function of $k \alpha H$ and $k^{2}$ (Stafford Smith et al 1982), and will be above the location of maximum beam shear. Obtaining the beam shear force from the maximum slope of the structure ignores the axial deformations in the walls thereby overestimating the shear force.

The maximum shear force in a coupling beam is obtained by integrating equation (21) and evaluating between $x+h / 2$ and $x-h / 2$. It can also be approximated as follows

$$
V_{\mathrm{b}, \max }=T_{\mathrm{csw}}^{\prime} h=\frac{q H h}{k^{2} \ell} \times \frac{E A c_{\mathrm{csw}}^{2}}{E A c^{2}} \times\left[K_{\mathrm{V}, \max }\right],
$$

in which a beam shear factor $K_{\mathrm{V} \text {, max }}$ represents the term in the square brackets of equation (21) and is to be evaluated at the location of maximum shear or can be obtained directly from the diagram in figure 5 .

The maximum chord rotation of a coupling beam can then simply be obtained as follows

$$
\theta_{\mathrm{b}, \max }=V_{\mathrm{b}, \max } \times \frac{b^{2}}{12 E I_{\mathrm{b}}}
$$

where $E I_{\mathrm{b}}$ is the bending stiffness of a coupling beam.

\subsection{Average shear force and increase of shear demand for critical coupling beam}

The average shear force in the coupling beams can be obtained from the maximum axial force in a shear wall and dividing through by the number of stories $(H / h)$ in the building. Setting $x=H$ and substituting into equation (15) yields an expression for the average shear force in the beams of a coupled wall bent

$$
\begin{aligned}
V_{\mathrm{b}, \mathrm{avg}}=\frac{T \ell_{\mathrm{x}=\mathrm{H}}}{H / h} & =\frac{q H h}{k^{2} \ell} \times \frac{E A c_{\mathrm{csw}}^{2}}{E A c^{2}}\left[\frac{1}{3}-\frac{1-(1 / k \alpha H-k \alpha H / 2) \sinh (k \alpha H)}{(k \alpha H)^{2} \cosh (k \alpha H)}\right] \\
& =\frac{q H h}{k^{2} \ell} \times \frac{E A c_{\mathrm{csw}}^{2}}{E A c^{2}} \times\left[K_{\mathrm{V}, \mathrm{avg}}\right]
\end{aligned}
$$

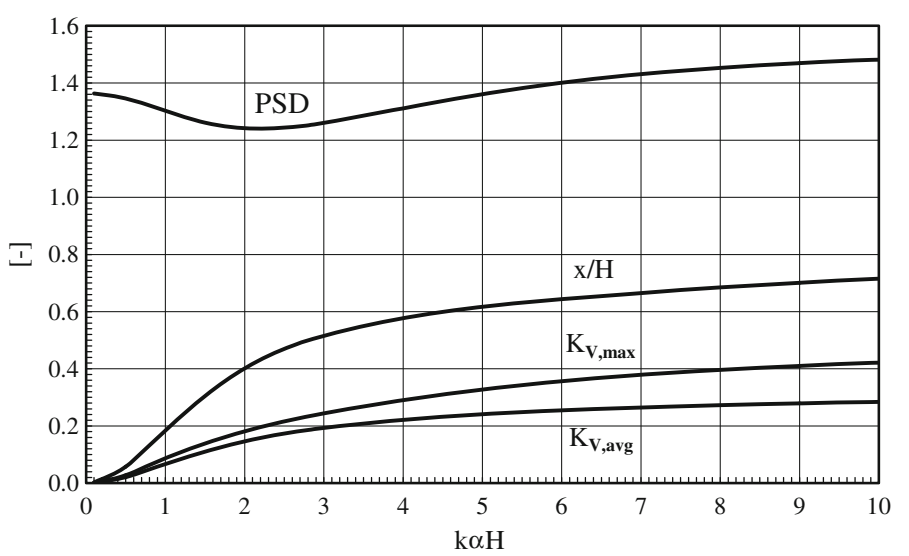

Figure 5. Design parameters for coupling beams. 
in which a factor for the average beam shear force $K_{\mathrm{V}}$,avg can be calculated or directly obtained from the diagram in figure 5. Equation (25) can also be used to obtain the average beam rotation $\theta_{\mathrm{b} \text {,avg }}$ by substituting $V_{\mathrm{b} \text {,avg }}$ for $V_{\mathrm{b} \text {,max }}$. The increase in shear demand for the critical coupling beam then is given by a peak shear demand factor which is defined as follows

$$
P S D=\frac{V_{\mathrm{b}, \max }}{V_{\mathrm{b}, \mathrm{avg}}}=\frac{K_{\mathrm{V}, \max }}{K_{\mathrm{V}, \mathrm{avg}}} .
$$

A curve in figure 5 represents the increase in shear demand for the critical coupling beams. This curve also represents the increase in rotational demand.

\section{Other loading cases}

Two other loading cases which may be required in practice are those of a uniformly distributed lateral load with intensity $w$ and a concentrated horizontal load $P$ at the top of the structure.

For a mixed shear wall structure subject to a uniformly distributed lateral load the constant $C$, to be used in equation (18) to obtain the $D o C$ of a coupled shear wall, is expressed as follows

$$
C_{\mathrm{UDL}}=2\left\{\frac{1-\cosh (k \alpha H)+(k \alpha H) \sinh (k \alpha H)}{(k \alpha H)^{2} \cosh (k \alpha H)}\right\} .
$$

The horizontal deflections up the height of the structure are given by

$$
\begin{aligned}
y_{\mathrm{UDL}}= & \frac{w H^{4}}{E I_{g}}\left[\frac{1}{8}-\frac{1}{6}\left(\frac{x}{H}\right)+\frac{1}{24}\left(\frac{x}{H}\right)^{4}+\frac{1}{k^{2}-1}\left\{\frac{1-(x / H)^{2}}{2(k \alpha H)^{2}}\right.\right. \\
& \left.\left.+\frac{\cosh (k \alpha H)(1-x / H)-1-(k \alpha H)[\sinh (k \alpha H)-\sinh (k \alpha x)]}{(k \alpha H)^{4} \cosh (k \alpha H)}\right\}\right] .
\end{aligned}
$$

The overturning moments are

$$
T \ell_{\mathrm{UDL}}=\frac{w H^{2}}{k^{2}}\left[\frac{1+(k \alpha x)^{2} / 2-\cosh (k \alpha x)}{(k \alpha H)^{2}}+\frac{[\sinh (k \alpha H)-(k \alpha H)] \sinh (k \alpha x)}{(k \alpha H)^{2} \cosh (k \alpha H)}\right] .
$$

For a mixed shear wall structure subject to a concentrated horizontal load at the top the constant $C$, to be used in equation (18) to obtain the $D o C$ of a coupled shear wall, is given by

$$
C_{\mathrm{PTL}}=\frac{\sinh (k \alpha H)}{(k \alpha H) \cosh (k \alpha H)} .
$$

The horizontal deflections up the height of the structure are

$$
y_{\mathrm{PTL}}=\frac{P H^{3}}{E I_{g}}\left[\frac{1}{3}-\frac{1}{2}\left(\frac{x}{H}\right)+\frac{1}{6}\left(\frac{x}{H}\right)^{3}+\frac{1}{k^{2}-1}\left\{\frac{1-(x / H)}{(k \alpha H)^{2}}+\frac{\sinh (k \alpha x)-\sinh (k \alpha H)}{(k \alpha H)^{3} \cosh (k \alpha H)}\right\}\right] .
$$

The overturning moments are

$$
T \ell_{\mathrm{PTL}}=\frac{P H}{k^{2}}\left[\frac{x}{H}-\frac{\sinh (k \alpha x)}{(k \alpha H) \cosh (k \alpha H)}\right] .
$$


For combinations of loading cases it is suggested that a weighted average of the degrees of coupling be used

$$
D o C=\frac{D o C_{\mathrm{PTL}}(P H)+D o C_{\mathrm{UDL}}\left(w H^{2} / 2\right)+D o C_{\mathrm{TDL}}\left(q H^{2} / 3\right)}{P H+w H^{2} / 2+q H^{2} / 3}=\frac{\sum\left(D o C \times M_{\mathrm{base}}\right)}{\sum\left(M_{\mathrm{base}}\right)} .
$$

Differentiating the equations for horizontal deflection and overturning moment allows expressions to be developed for maximum shear in and rotation of the coupling beams in addition to their location up the height of the structure. This will also yield the peak shear and rotation demands.

\section{Example}

\subsection{General data}

The floor plan of a mixed shear wall structure is shown in figure 6. It comprises three coupled shear wall bents (CSW) and two single planar shear walls (SSW) at the opposite ends of the building. The analysis of this structure will be compared to a coupled wall structure with five identical coupled wall bents on the same floor layout, i.e., the end walls are replaced by coupled walls. The structure has a total height of $60 \mathrm{~m}$ and consists of 20 stories of equal height. For all analyses the lateral loading and maximum deflection at the top of the structure are kept constant. The maximum intensity of the triangularly distributed lateral load at the top of the structure is $4.0 \mathrm{kN} / \mathrm{m}^{2}$ and the modulus of elasticity for the concrete is $27 \mathrm{kN} / \mathrm{mm}^{2}$. This yields a sway of

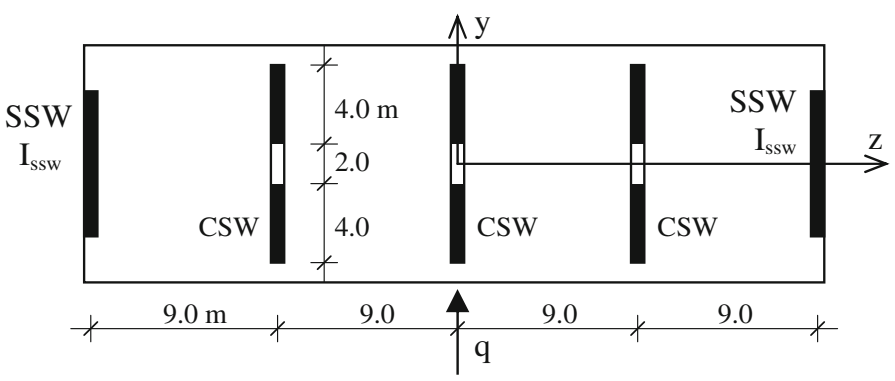

Figure 6. Floor plan with three coupled wall bents and single planar end walls.

Table 1. Characteristic parameters for coupled shear wall structure.

\begin{tabular}{|c|c|c|c|c|c|c|c|c|c|}
\hline \multirow[b]{3}{*}{ Structure } & \multirow{3}{*}{$\begin{array}{l}\text { Beam } \\
\text { depth } \\
\mathrm{d}, \mathrm{m}\end{array}$} & \multirow{3}{*}{$\begin{array}{c}\text { Wall and } \\
\text { beam thickness } \\
\mathrm{t}, \mathrm{m}\end{array}$} & \multirow[t]{3}{*}{$\mathrm{k}^{2}$} & \multirow{3}{*}{$\begin{array}{r}\mathrm{k} \alpha \mathrm{H} \\
(4)\end{array}$} & \multirow{3}{*}{$\begin{array}{c}\text { DoC }_{\mathrm{csw}} \\
(1) \\
\text { Fig. } 2\end{array}$} & \multirow{3}{*}{$\begin{array}{c}\mathrm{x} / \mathrm{H} @ \mathrm{~V}_{\mathrm{b}, \max } \\
(23) \\
\text { Fig. } 5\end{array}$} & \multirow{3}{*}{$\begin{array}{c}\mathrm{V}_{\mathrm{b}, \max } \mathrm{kN} \\
(24)\end{array}$} & \multirow{3}{*}{$\begin{array}{c}\text { PSD } \\
(27) \\
\text { Fig. } 5\end{array}$} & \multirow{3}{*}{$\begin{array}{c}\theta_{\mathrm{b}, \max } . \\
(25)\end{array}$} \\
\hline & & & & & & & & & \\
\hline & & & & & & & & & \\
\hline CSW1 & 020 & 00 & 1.104 & 1.6 & (202 & 220 & 117 & 1.26 & 0006 \\
\hline CSW2 & 0.30 & & & 2.93 & & & & 1.26 & 0.00567 \\
\hline CSW3 & 0.40 & 0.3 & 1.104 & 4. & & & 243 & 1.34 & 0.00469 \\
\hline CSW4 & 0.50 & 0.2 & 1.104 & 6.30 & 0 . & & 285 & 1.41 & 0.00365 \\
\hline CSW5 & 0.60 & 0.194 & 1.104 & 8.28 & 0.747 & 0.689 & 313 & 1.46 & 0.00277 \\
\hline
\end{tabular}




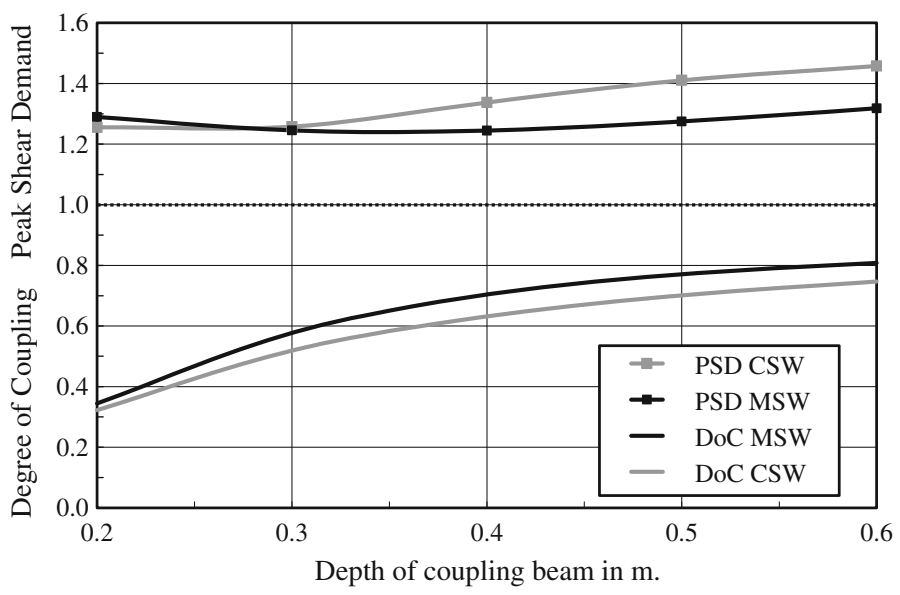

Figure 7. Influence of coupling beam size on DoC and PSD.

$1 / 513(\approx 0.2 \%)$ for all structures. The width of the coupled walls and the length of the coupling beams are shown in figure 6 . The effective bending stiffness of the coupling beams is taken to be $0.4 E I_{\mathrm{b}}$ and for the shear walls $0.7 E I_{\mathrm{csw}}$.

\subsection{Coupled shear wall structure}

In the CSW structure the beam depth of the five identical coupled wall bents will be increased step-wise from $0.2 \mathrm{~m}$. to $0.6 \mathrm{~m}$. In order to arrive at the same horizontal deflection at the top, the thickness of the walls and beams are adjusted as necessary. Table 1 shows the changes in the characteristic parameters of the coupled wall structure. An increase in beam depth increases the characteristic parameter $k \alpha H$, the degree of coupling, DoC and peak shear demand, PSD. Although the peak coupling beam rotation decreases with increasing beam depth, the peak rotation demand is identical to its peak shear demand. This is also clearly shown in the diagram of figure 7 where the grey curves for the CSW structures display these trends. It should be noted that the values for $k^{2}$ remain unchanged when the wall thicknesses are adjusted.

\subsection{Mixed shear wall structure}

In the MSW structure as shown in figure 6 the depth of the coupling beams are also increased step-wise from 0.2 to $0.6 \mathrm{~m}$. The thickness of the walls and beams are identical to those of the

Table 2. Characteristic parameters for mixed shear wall structure.

\begin{tabular}{|c|c|c|c|c|c|c|c|c|c|}
\hline & Beam & Single & $\mathrm{k}^{2}$ & $\mathrm{k} \alpha \mathrm{H}$ & DoC & $\mathrm{x} / \mathrm{H} @ \mathrm{~V}_{\mathrm{b} \text {.max }}$ & $\mathrm{V}_{\mathrm{b}, \text { max }}$ & PSD & $\theta_{\mathrm{b}, \max }$ \\
\hline Structure & $\begin{array}{l}\text { depth } \\
\mathrm{d}, \mathrm{m}\end{array}$ & $\begin{array}{c}\text { shear walls } \\
\mathrm{I}_{\mathrm{ssw}}, \mathrm{m}^{4}\end{array}$ & (6) & (4) & $\begin{array}{l}\text { (18) } \\
\text { Fig. } 4\end{array}$ & $\begin{array}{l}\text { (23) } \\
\text { Fig. } 5\end{array}$ & $\begin{array}{c}\text { (24) } \\
\text { Fig. } 5\end{array}$ & $\begin{array}{c}\text { (27) } \\
\text { Fig. } 5\end{array}$ & (25) \\
\hline MSW1 & 0.20 & 21.5 & 1.228 & 1.14 & 0.345 & 0.219 & 120 & 1.29 & 0.00690 \\
\hline MSW2 & 0.30 & 21.0 & 1.318 & 1.83 & 0.578 & 0.373 & 185 & 1.25 & 0.00557 \\
\hline MSW3 & 0.40 & 20.6 & 1.422 & 2.54 & 0.704 & 0.473 & 220 & 1.24 & 0.00425 \\
\hline MSW4 & 0.50 & 20.7 & 1.520 & 3.30 & 0.771 & 0.538 & 245 & 1.27 & 0.00317 \\
\hline MSW5 & 0.60 & 20.9 & 1.602 & 4.14 & 0.808 & 0.584 & 267 & 1.32 & 0.00236 \\
\hline
\end{tabular}


Table 3. Changes between CSW and MSW structures.

\begin{tabular}{lcc}
\hline $\begin{array}{l}\text { Beam depth } \\
\mathrm{d}, \mathrm{m}\end{array}$ & $\begin{array}{c}\text { DoC } \\
\text { change, } \%\end{array}$ & $\begin{array}{c}\text { PSD } \\
\text { change, } \%\end{array}$ \\
\hline 0.20 & 6.90 & 2.74 \\
0.30 & 11.28 & -0.98 \\
0.40 & 11.47 & -6.95 \\
0.50 & 9.94 & -9.66 \\
0.60 & 8.28 & -9.57 \\
\hline
\end{tabular}

CWS structures. In order to obtain the same horizontal deflection at the top of the structures, only the second moment of area of the single planar shear walls are adjusted. Table 2 shows the changes in the characteristic parameters of the mixed shear wall structure. An increase in beam depth increases both characteristic parameters $k^{2}$ and $k \alpha H$. It also increases the $D o C$ but leaves the $P S D$ almost unchanged. This is also shown in figure 7, where the black curves for the MSW structures display these trends.

\subsection{CSW versus $M S W$}

The curves in figure 7 clearly show that replacing two CSW bents by two SSW's has a significant influence on the behaviour of the coupled walls and the connecting beams. Keeping the overall lateral stiffness of the structure unchanged, it increased the characteristic parameter $k^{2}$ and decreased $k \alpha H$. Replacing two coupled wall bents by two single shear walls have

- increased the $D o C$ and peak shear force in the beam $V_{\mathrm{b}, \max }$,

- decreased the PSD and peak chord rotation of the beam $\theta_{\mathrm{b}, \max }$ and

- moved the location of peak beam shear and rotation up the height of the structure.

Furthermore, it is shown in table 3 that a maximum increase in $D o C$ does not result in a maximum decrease in $P S D$.

\section{Conclusions}

A simplified method of analysis to obtain the degree of coupling, DoC for coupled wall bents and peak shear demand, PSD for the critical coupling beams in mixed shear wall structures subject to triangularly distributed horizontal loading, has been presented. The proposed design procedure also yields estimates for the maximum shear force in and maximum rotation of the coupling beams in addition to their location up the height of the structure. Diagrams were given for these design parameters. The analysis is based on the continuous medium theory as applied to coupled wall structures and therefore only applicable to high-rise structures. This theory allows a MSW structure to be represented by two characteristic non-dimensional parameters, $k^{2}$ and $k \alpha H$, which define its overall structural behaviour.

The parameter $k \alpha H$ allows rapid graphical assessments of five important design parameters for the coupling beams: peak shear demand, peak rotation demand, maximum shear force, maximum rotation and their location up the height of the structure.

In general, an increase in $k \alpha H$ :

- increases the peak shear force in the coupling beam,

- decreases the beam rotation, 
- moves the location of peak shear and rotation downward to the base of the structure and

- will increase the peak shear and rotation demands for $k \alpha H>2$.

The range in peak shear and peak rotation demand factors is between 1.24 and 1.52. The method of analysis is restricted to structures in which the structural floor plan arrangement is symmetric. The theory is based on the assumption of, and therefore is accurate only for, structures that are uniform through their height. It may be used, however, to obtain approximate values for the design parameters for comparison between practical structures whose properties vary with height.

\section{References}

Chaallal O, Gauthier D and Malenfant P 1996 Classification methodology for coupled shear walls. ASCE J. Struct. Eng. 122(12): 1453-1458

Chitty L 1947 On the cantilever composed of a number of parallel beams interconnected by cross-bars. Philosophical Mag. 7(38): 685-699

Coull A and Choudhury J R 1967 Stresses and deflections in coupled shear walls. Proc. Am. Concrete Inst. 64(2): 65-72

Harries K A, Moulton J D and Clemson R L 2004 Parametric study of coupled wall behaviour - implications for the design of coupling beams. ASCE J. Struct. Eng. 130(3): 480-488

Hoenderkamp J C D and Stafford Smith B 1984 Simplified analysis of symmetric tall building structures subject to lateral loads. In: Y K Cheung and P K K Lee (eds.), Proceedings of the Third International Conference on Tall Buildings. Hong Kong, Guangzhou, pp. 28-36

Stafford Smith B and Coull A 1991 Tall building structures - analysis and design, New York: Wiley Interscience

Stafford Smith B, Kuster M and Hoenderkamp J C D 1981 A generalized approach to the deflection analysis of braced frame, rigid frame and coupled wall structures. Can. J. Civil Eng. 8: 230-240

Stafford Smith B, Hoenderkamp J C D and Kuster M 1982 A graphical method of comparing the sway resistances of tall building structures. Proceedings Institution of Civil Engineers, Part 2. 73: 713-729

Stafford Smith B, Hoenderkamp J C D and Kuster M 1984 Generalized method for estimating the drift in high-rise structures. ASCE J. Struct. Eng. 110(7): 1549-1562 